\title{
An Introduction to the EcoTroph $R$ Package: Analyzing Aquatic Ecosystem Trophic Networks
}

\author{
by Mathieu Colléter, Jérôme Guitton and Didier Gascuel
}

\begin{abstract}
Recent advances in aquatic ecosystem modelling have particularly focused on trophic network analysis through trophodynamic models. We present here a $\mathrm{R}$ package devoted to a recently developed model, EcoTroph. This model enables the analysis of aquatic ecological networks and the related impacts of fisheries. It was available through a plug-in in the well-known Ecopath with Ecosim software or through implementations in Excel sheets. The R package we developed simplifies the access to the EcoTroph model and offers a new interfacing between two widely used software, Ecopath and R.
\end{abstract}

\section{Introduction}

In the face of the global overexploitation of marine resources and the fast degradation of ecosystems integrity, scientists developed new modelling approaches at the scale of the ecosystem. In fact, the main tool used for fisheries regulation is a stock approach which does not account for the trophic network linking marine ecological components. An important challenge is to analyze the aquatic ecological networks and the related impacts of fishery. There are several ways to define and represent an ecosystem. One widely used approach is based on the trophic level concept. Elton (1927) and Lindeman (1942) introduced this concept for describing aquatic ecosystems by assigning integer trophic levels (TLs) to the individual numbers, to the biomass or to the biological production by its component species. This approach differentiated between primary producers and detritus (TL $=1)$, first-order consumers $(\mathrm{TL}=2)$, second-order consumers $(\mathrm{TL}=3) \ldots$ The ecosystem is so represented as a pyramid of number, biomass or production, from low to high TLs. Odum and Heald (1975) developed this concept by implementing fractional trophic levels resulting from the diet of the individual and the trophic level of its preys. The emergence of Ecopath as a widely used approach and software for modelling aquatic ecosystems (Polovina, 1984; Christensen and Pauly, 1992) contributed in a major way to the prominence of TLs, especially as they were not an input, but an output of the model (i.e. estimated parameters). As the use of Ecopath spread worldwide with hundreds of application cases, so did the trophic level concept.

EcoTroph (ET) is an approach and software for modelling marine and freshwater ecosystems, entirely articulated around the TL concept (Gascuel, 2005; Gascuel and Pauly, 2009). It has been developed at the same time as the Ecopath worldwide expansion happened and incorporated into the Ecopath plug-in family (Gascuel et al., 2009). The first key idea of ET is that it deals with the continuous distribution of the biomass in an ecosystem as a function of continuous TL. The biomass enters the foodweb at TL $=1$, generated by the photosynthetic activity of primary producers, or recycled from the detritus by the microbial loop. Between TL $=1$ and TL $=2$, the biomass is composed of mixotrophs only, and is usually low. If any, it is conventionally split between biomasses at TL $=1$ and 2 . Then, at TLs $\geq 2$, the biomass is composed by heterotrophic organisms with mixed diet and fractional TLs resulting in a continuous distribution of biomass along TLs. The second key feature of ET is that the trophic functioning of aquatic ecosystems is modelled as a continuous flow of biomass surging up the foodweb, from lower to higher TLs, through predation and ontogenic processes. All the equations of the model are detailed in Gascuel et al. (2011). Such an approach, wherein species as such disappear, may be viewed as the final stage in the use of the TL metric for ecosystem modelling. It provides a simplified but useful representation of ecosystem functioning and impact of fishing. Thus, ET has been used both in theoretical contexts based on virtual ecosystems (Gascuel and Pauly, 2009; Gascuel et al., 2011), or in specific case studies to assess the current fishing impacts at the ecosystem scale (Gasche et al., 2012, in the South African Benguela ecosystem, Lassalle et al., 2012, in the Bay of Biscay, Tremblay-Boyer et al., 2011, for a worldwide analysis), or to analyze the effects of marine protected areas on the whole food web (Colléter et al., 2012 in Sénégal, Valls et al., 2012 in the Mediterranean Sea). Furthermore, ET enables the construction of a unique comparison framework for Ecopath models, the trophic spectrum. This display, based on ET key ideas, is a graphical representation of the ecosystem parameters, such as biomass, production, catch, fishing mortality, etc., along the trophic level (Gascuel et al., 2005). Examples of the use of the ET model and the associated trophic spectra analyses are provided in Figure 1.

This package is the first attempt to offer interfacing between $\mathrm{R}$ and Ecopath through its plug-in 


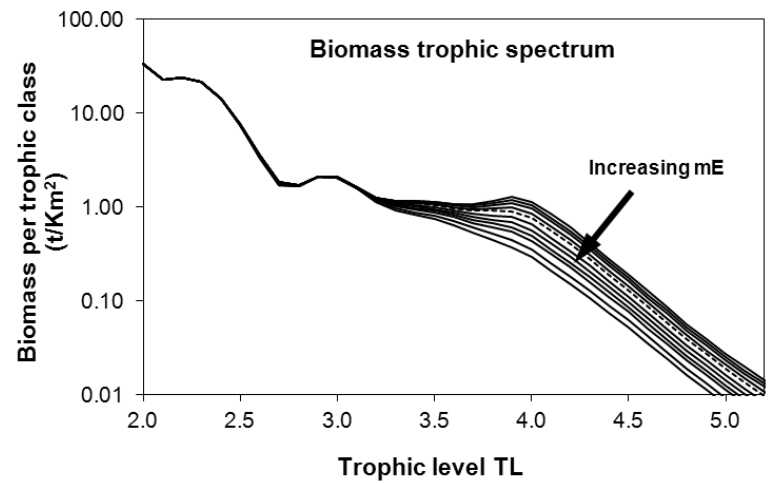

(a) Fishing impact on biomass in the Guinean ecosystem. Simulations refer to various multipliers (mE) of the current (i.e. 2004) fishing mortalities, from $\mathrm{mE}=0$ (i.e. virgin state) to $\mathrm{mE}=5$. Results highlight the strong impact of the current fishing effort ( $\mathrm{mE}=1$, dashed lines) on the high TLs biomass: biomass is divided by 2 compared to the virgin state for all trophic levels higher than 3.8. In contrast, TLs around 3 benefit from a release of predation which approximately counterbalances the fishing impact.

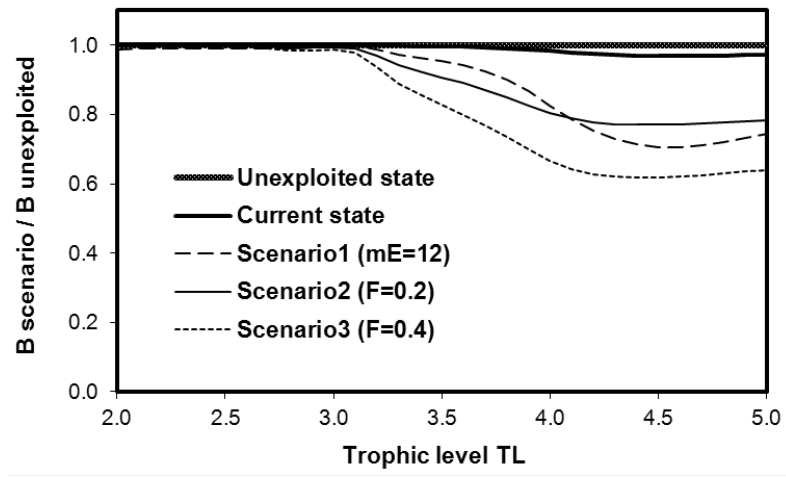

(c) Diagnosis on the effect of restricting fishery in the Port-Cros Marine Protected Area (France, Mediterranean Sea): trophic spectra of the relative biomass for a total closure of the fishery and 3 other hypothetical fishing scenarios. Results show that the MPA fulfills its conservation objective with current biomass very close to the virgin state for all trophic levels. Conversely, the other fishing scenarios lead to a significant decrease in the biomass of high trophic levels. It was concluded that the MPA mostly benefits predators and the functional trophic biodiversity.

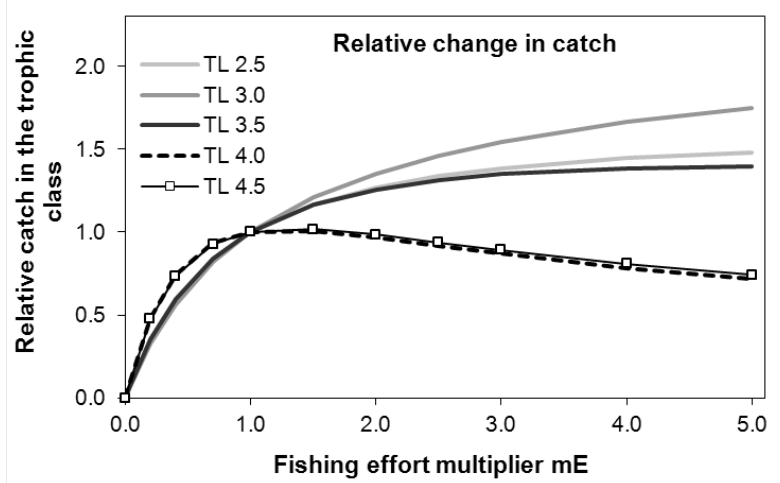

(b) Diagnosis on catch made in the Guinean ecosystem. Relative values of catch simulated for some trophic classes are expressed as a function of multipliers of the current fishing mortalities. Results show that high TLs (TL $=4$ and 4.5 ) are fully exploited as an increase in the fishing pressure leads to a decrease in catch. In contrast, yields could be increased for the lower TLs. In other words, increasing fishing pressure would lead to a decrease in the mean trophic level of catch.

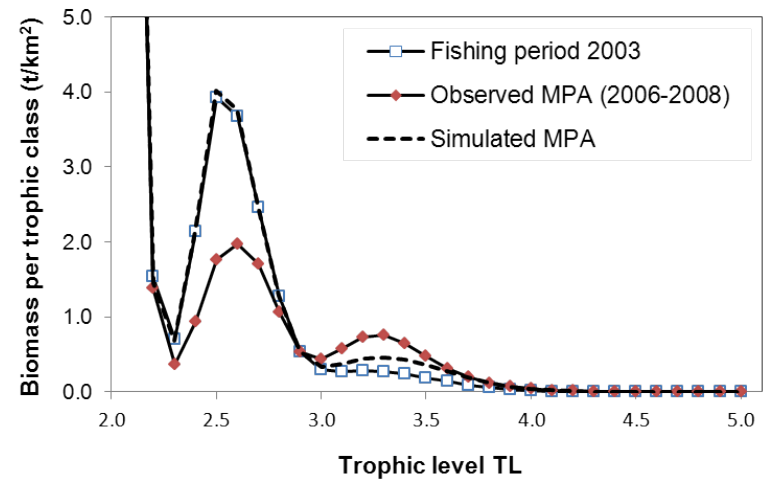

(d) Analysis of the Marine Protected Area effect in the Bolong de Bamboung (Sénégal). Using the ETTranspose routine, biomass trophic spectra are built from the 2003 Ecopath model (before the closure of the fishery) and the 2006-2008 model (enforced closure). The ET-Diagnosis routine is used to simulate the MPA effect, starting from the 2003 model and applying a null fishing mortality. Results show that the closure of the fishery can explain, at least partially, the increase observed in the biomass of high TLs $(\geq 3.3)$. The decrease observed for intermediate TLs may not only results from a release in predation. Behavioral (refuge, flee) and environmental effects, that are not included in the model, may thus explain the observed differences.

Figure 1: Examples of the use of the EcoTroph model: diagnosis on the fishing impact on biomass (a) and catches (b) in the Guinean Ecosystem (from Gascuel et al, 2011); assessment of a Marine Protected Area (MPA) in Port-Cros, France (c) (from Valls et al, 2012) and in the Bolong of Bamboung, Sénégal (d) (from Colléter et al., 2012). 
EcoTroph. The plug-in will use $\mathrm{R}$ and the EcoTroph (Guitton et al., 2013) package within the free Ecopath with Ecosim software. This enables the use of ET for a large panel through the plug-in for inexperienced $\mathrm{R}$ users, or directly the $\mathrm{R}$ package for the more expert ones. On the developer side, this way to link a rich user interface (developed in Microsoft VB) and a well known software in the fishery scientists group such as $\mathrm{R}$ is a way to set up a community. These researchers can so focus on the model improvements without taking into account the rich user interface which is time consuming. Along with several functions, we include an example dataset on the Guinean marine ecosystem (Gascuel et al., 2011) within the package. This article introduces, using the example dataset, the three main components of EcoTroph:

1. The data import and validation

2. The ET-Transpose tool

3. The ET-Diagnosis tool

A great deal of documentation, both introductory and advanced, is available on the ET website (http://sirs. agrocampus-ouest.fr/EcoTroph). The EcoTroph package requires the XML package (Lang, 2012) in order to load the model input parameters coming from the Ecopath software. The read. ecopath. model function will parse the data.

\section{Data import and validation}

The EcoTroph package requires an input data table to run. The user has to load this dataset under the different possible formats ('.xls', '.csv', '.txt') with the functions read. table, read.csv... The ecopath_guinee dataset (Table 1 ) is an example of a suitable input table constructed for use with the EcoTroph package.

The variable names have to be specified and strictly the same as above: group_name (name of the group representing one or several species gathered together), TL (the trophic level of the group), biomass (the biomass of the group), prod (the production on biomass ratio or P/B) and accessibility (the ratio of the group biomass that would be caught assuming an infinite fishing pressure). These parameters generally come from Ecopath inputs or outputs, but can also be independent. The entry catch.1, catch.2, catch. whatyouwant is necessary if several fisheries do exist. The OI column (the omnivory index, an Ecopath output parameter) is optional, it is used in the create. smooth function for an alternative smooth form (sigmaLN $=\frac{O I}{T L_{j}}$, see below).

The check. table function was developed to check the compatibility of the input data table with the EcoTroph package:

check.table(ecopath_guinee)

In the example, no warning message appears as no error is made. If a message appears, the user has to correct the dataset in view of the comments. No missing values (NAs) are accepted as input, a yield column has to be entered with 0 -values if no catches are registered. As well as the yield, the P/B of detritus groups (not entered in Ecopath) has to be set to 0 . The check. table function converts the input dataset into a "data. frame" object if it is not already one.

A read.ecopath.model function was also implemented. It allows users to import data in '.xml' format exported from the Ecopath/EcoTroph plug-in or distributed by a web service (a database of Ecopath models has been set up to allow a meta-analysis at a world scale, http://sirs. agrocampusouest.fr/EcoTroph). This function formats the inputs so they meet the EcoTroph package requirements. Nevertheless, it is advisable to check the data consistency using the check. table function.

\section{ET-Transpose tool}

\section{Creation of the Smooth function}

The Smooth function returns a table allowing the conversion of data referring to specific taxons or functional groups (Ecopath trophic groups for example) into data referring to trophic classes. The major assumption of this function is that distributions of the trophic groups' biomass, yield, etc., around their mean trophic level follow a lognormal curve using the equation:

$$
P_{i j}=\frac{1}{\left(\mathrm{TL}_{i}-\text { shift }\right) \sigma_{j} \sqrt{2 \pi}} \exp \left[-\frac{\left(\ln \left(\mathrm{TL}_{i}-\text { shift }\right)-\ln \left(\overline{\mathrm{TL}_{j}}-\text { shift }\right)\right)^{2}}{2 \sigma_{j}^{2}}\right]
$$




\begin{tabular}{lllrlllll}
\hline & group_name & TL & biomass & prod & catch.1 & catch.2 & accessibility & OI \\
\hline 1 & Whales & 4.01 & 0.0309 & 0.020 & 0.000 & 0.000 & 0.0 & 0.059 \\
2 & Dolphins & 4.48 & 0.0433 & 0.070 & 0.000 & 0.000 & 0.0 & 0.331 \\
3 & Turtles & 2.19 & 0.0296 & 0.150 & 0.000 & 0.000 & 0.0 & 0.338 \\
4 & Sea birds & 3.81 & 0.0013 & 0.300 & 0.000 & 0.000 & 0.0 & 0.353 \\
5 & Rays+ & 3.97 & 0.3860 & 0.363 & 0.012 & 0.024 & 0.9 & 0.329 \\
6 & Sharks+ & 4.31 & 0.1050 & 0.410 & 0.007 & 0.003 & 0.8 & 0.633 \\
7 & Large pelagics & 4.21 & 0.3840 & 0.850 & 0.025 & 0.069 & 0.8 & 0.263 \\
8 & Barracudas+ & 4.12 & 0.0583 & 0.920 & 0.009 & 0.022 & 0.9 & 0.259 \\
9 & Carangids & 4.16 & 0.0627 & 1.000 & 0.010 & 0.024 & 0.8 & 0.139 \\
10 & Horse Makerels+ & 3.13 & 2.3330 & 0.700 & 0.000 & 0.115 & 0.8 & 0.366 \\
& $\ldots$ & $\ldots$ & $\ldots$ & $\ldots$ & $\ldots$ & $\ldots$ & $\ldots$ & $\ldots$ \\
34 & Primary producers & 1.00 & 69.0000 & 84.000 & 0.000 & 0.000 & 0.0 & 0.000 \\
35 & Detritus & 1.00 & 290.0000 & 0.000 & 0.000 & 0.000 & 0.0 & 0.193 \\
\hline
\end{tabular}

Table 1: Data from the Ecopath model of the Guinean ecosystem (extracts).

The lognormal distribution is defined by: a mean (the mean trophic level of the group, $\overline{\mathrm{TL}}_{j}$ ), a standard deviation ( $\sigma_{j}$ denoted as sigmaLN in the $\mathrm{R}$ code) which is a measure of the trophic level variability within the group, and a shift parameter defining the theoretical trophic level characterised by a null variability in TL within group.

The create. smooth function enables the creation of this Smooth function using several input parameters. The parameter ecopath corresponds to the input data table (ecopath_guinee in the example). The parameter pas defining the splitting of trophic classes has by default a value of 0.1 . The parameter smooth_type defines the form of the standard deviation (sigmaLN) wanted for the lognormal distribution. Three options are implemented:

1. If smooth_type=1 (choice by default), sigmaLN is constant. This constant sigmaLN is equal to the parameter sigmaLN_cst specified in the function, and has by default a value of 0.12 . The shift parameter is set equal to 1.8 by default.

2. If smooth_type $=2$, this is equivalent to sigmaLN=smooth_param $* \ln (T L-0.05)$. The parameter smooth_param of this formula (also a parameter of the create.smooth function) defines the slope of the log-linear trophic level variability increase around the mean trophic level of the group. Based on our experience gained partially through observations, default parameters have been defined as follows: smooth_param $=0.07$ and shift $=0.95$. (No need to change the shift value in the function, let shift=NULL, it will be automatically set to 0.95 for smooth_type=2. Same thing for the parameter smooth_param.)

3. If smooth_type $=3$, sigmaLN for each group is equal to the omnivory index calculated by Ecopath divided by the mean trophic level of the group. A warning message will appear if OIs are equal to 0 , they will be automatically changed to a value of 0.01 . The parameter shift is by default equal to 0 . (No need to change the shift value in the function, let shift=NULL, it will be automatically set to 0 for smooth_type=3.)

The create. smooth function returns a table of the distribution of each mean trophic level within trophic classes (i.e. how a given species or ecological group, characterized by a given and known mean trophic level, is distributed around this trophic level). This table will be used in the next step of the analysis for the construction of trophic spectra.

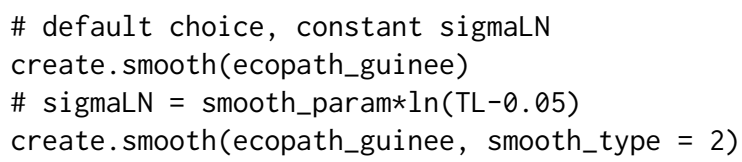

A graphic function, plot (smooth), was developed in order to display this Smooth function. The input parameter is the table returned by the create.smooth function. It returns a plot with the lognormal curve for each present trophic class (see Figure 2).

plot (create.smooth(ecopath_guinee)) 


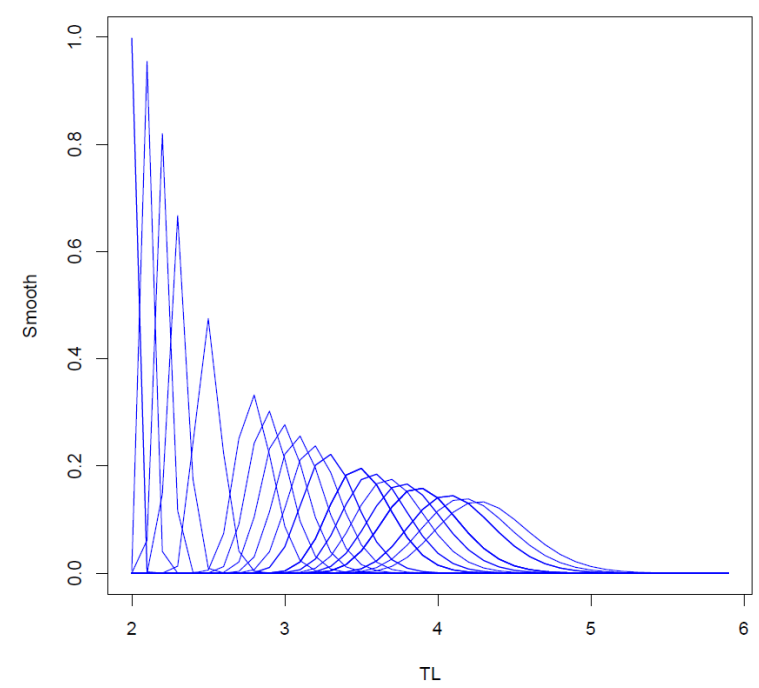

Figure 2: Output of the plot (smooth) graphic function, applied to the Guinean ecosystem example. Each curve represents the distribution across trophic levels for all groups whose mean trophic level is equal to the mean value of the distribution. Only curves related to existing groups are displayed.

\section{Data transposition}

The Transpose function enables the conversion of data referring to specific taxons or functional groups (Ecopath trophic groups for example) into data referring to trophic classes. This function uses the table returned by the create. smooth function. The concerned variables are the biomasses, or the catches or others ... Using the Transpose function, these variables are distributed continuously along the trophic classes for each group. This function will be reused in the create. ETmain function to build a summary table with all the variables calculated by trophic class.

Transpose takes as input parameters the table returned by the create.smooth function (tab_smooth), the input data table (ecopath), and the name of the column the user wants to distribute by trophic class (column):

A $<-$ create.smooth(ecopath_guinee)

\# Transpose of the biomass column

T_biomass <- Transpose(A, ecopath_guinee, "biomass")

\# Transpose of the catch.1 column

Transpose(A, ecopath_guinee, "catch.1")

Results can be displayed graphically using the plot(Transpose) function. It takes as input parameter the table returned by the Transpose function (tab_Trans). The user has the possibility to use a $\log$ scale for the $y$-axis ( $\mathrm{scale}=\log$, the minimum value considered on the graph is conventionally set up at 1/10000 of the total biomass), and to enter a title (title):

plot $($ tab_Trans, title $=$ NULL, scale $=$ NULL) returns the principal plots according to the selected column, in particular a plot by group and the associated trophic spectra (see Figure 3).

\# title and log scale for the biomass

plot(T_biomass, title = "biomass", log)

\section{ET_Main table creation}

The create. ETmain function enables the creation of a summary table, ET_Main, containing the principal variables by trophic class. This function can be used directly, there is no need of the previous steps Smooth and Transpose (however these steps are necessary to a good understanding of the EcoTroph model). It takes as input parameters the input data table (ecopath) and the parameters of the create. smooth function:

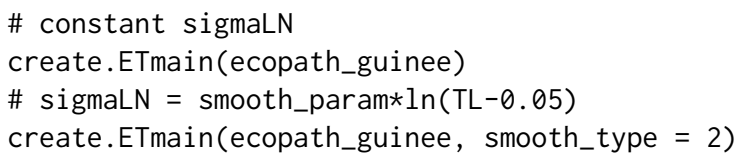



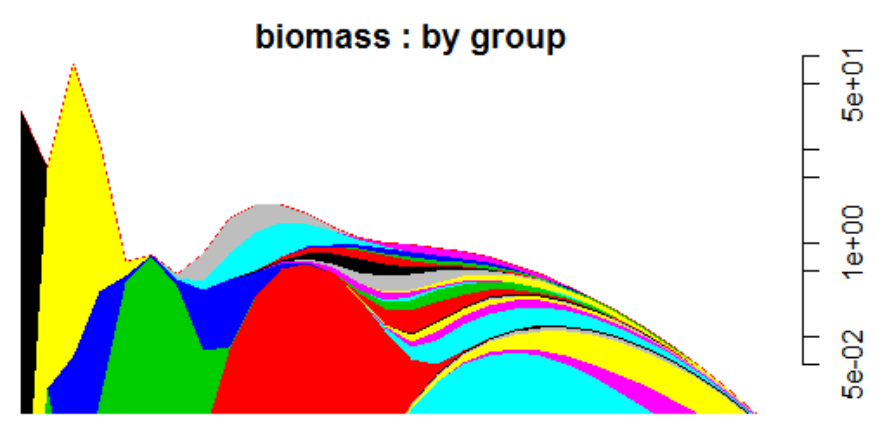

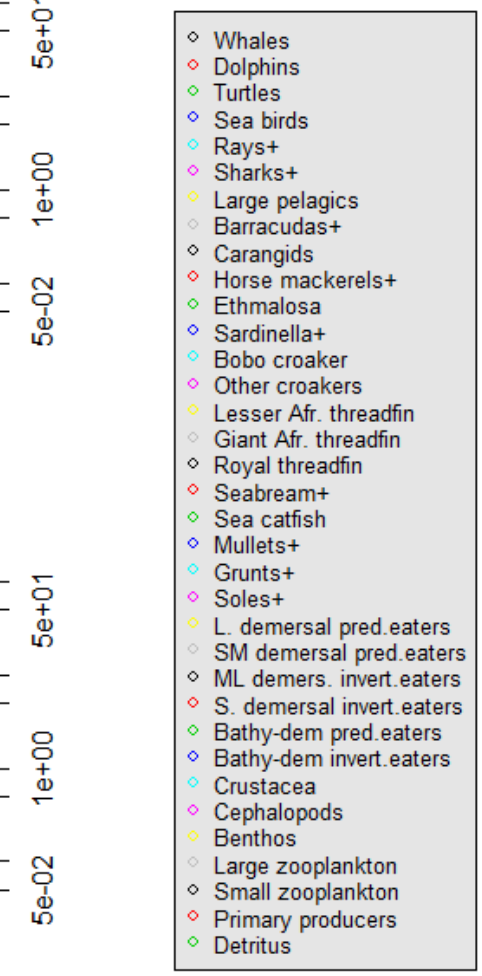

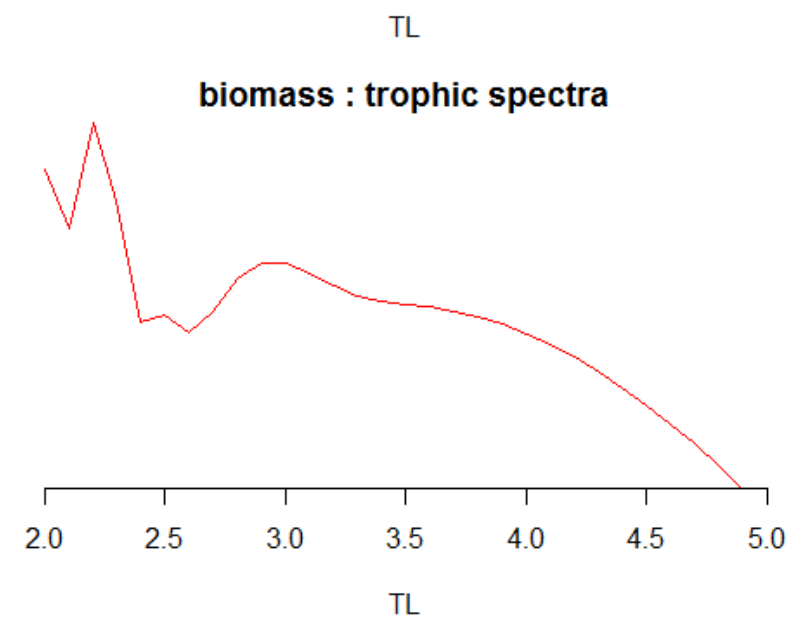

Figure 3: Output of the plot (Transpose) graphical function applied to the biomass data per ecological group in the Guinean ecosystem (ecopath_guinee dataset). The top panel displays the distributions for each group, while the bottom panel displays the biomass trophic spectrum (BTS, i.e. the sum of all groups). 


\begin{tabular}{lrlrlllll}
\hline TL & \multicolumn{1}{l}{ B } & B_acc & P & P_acc & Kin & Kin_acc & Y_tot & F_loss \\
\hline 1 & 359.00 & 0.00 & 5796.00 & 0.00 & 16.14 & 1.00 & 0.00 & 0.00 \\
2 & 25.73 & 0.00 & 1029.20 & 0.00 & 40.00 & 0.68 & 0.00 & 0.00 \\
2.1 & 6.26 & 0.00 & 13.40 & 0.00 & 2.14 & 0.68 & 0.00 & 0.00 \\
2.2 & 82.06 & 0.06 & 147.64 & 0.04 & 1.80 & 0.68 & 0.01 & 0.00 \\
2.3 & 11.94 & 0.27 & 21.17 & 0.20 & 1.77 & 0.74 & 0.05 & 0.00 \\
2.4 & \multicolumn{1}{c}{0.62} & 0.44 & 0.90 & 0.57 & 1.44 & 1.31 & 0.24 & 0.00 \\
& $\ldots$ & $\ldots$ & $\ldots$ & $\ldots$ & $\ldots$ & $\ldots$ & $\ldots$ & $\ldots$ \\
\hline
\end{tabular}

\begin{tabular}{llrllllc}
\hline TL & F_loss_acc & N_loss & Fish_mort & Fish_mort_acc & Selec & Time & N_loss_acc \\
\hline 1 & 0.00 & 1.73 & 0.00 & 0.00 & 0.00 & 0.00 & NaN \\
2 & 0.22 & 43.41 & 0.00 & 0.15 & 0.00 & 0.06 & -197.08 \\
2.1 & 0.22 & -24.00 & 0.00 & 0.15 & 0.00 & 0.06 & -70.66 \\
2.2 & 0.22 & 19.42 & 0.00 & 0.15 & 0.00 & 0.11 & -16.80 \\
2.3 & 0.25 & 31.57 & 0.00 & 0.18 & 0.02 & 0.17 & -10.82 \\
2.4 & 0.42 & -1.89 & 0.38 & 0.55 & 0.70 & 0.22 & -6.45 \\
& $\ldots$ & $\ldots$ & $\ldots$ & $\ldots$ & $\ldots$ & $\ldots$ & $\ldots$ \\
\hline
\end{tabular}

Table 2: create.ETmain(ecopath_guinee)\$ET_Main results (extracts).

In the ET_Main output table (Table 2), some parameters directly come from the input table (biomass B, catch Y_tot), while some are calculated based on the definitions of EcoTroph parameters (e.g. Fishing mortality Fish_mort $=Y / B$, fishing loss rate $F \_l o s s=Y / P$, see details in Gascuel et al., 2009).

\# constant sigmaLN

ET_Main <- create.ETmain(ecopath_guinee) \\$ET_Main

ET_Main

The create.ETmain function also returns intermediate tables, i.e. the following tables are also contained in the returned list object:

. biomass, the table returned by the Transpose function for the column biomass.

. biomass_acc, the table biomass multiplied by the accessibility parameter.

- prod, the table corresponding to the production by trophic class.

- prod_acc, the table prod multiplied by the accessibility parameter.

. tab_smooth, the table returned by the create. smooth function.

. $Y$, the table(s) returned by the Transpose function for the column catch. ( $x$ ).

$>$ names(create.ETmain(ecopath_guinee))

[1] "ET_Main" "biomass" "biomass_acc" "prod" "prod_acc" "tab_smooth" "Y"

As previously, we developed a graphic function to display the main results: plot (ETmain). Different plots are created, e.g. the biomass trophic spectrum (BTS), the accessible biomass trophic spectrum (ABTS), the catch trophic spectrum (CTS). It takes as input parameter the list object returned by the create. ETmain function.

plot (create.ETmain (ecopath_guinee), log)

Naturally all the returned graphics are not exhaustive. The user can construct other ones using the returned list object. Moreover the plot(ETmain) function is implemented with a log scale parameter for the different trophic spectra. However, this does not always provide a good representation. We really encourage users to test different scales for the $y$-axis. Some plots could be falsely interpreted with no awareness of the $y$-axis form importance.

\section{ET-Diagnosis simulation tool}

ET-Diagnosis is used to simulate the effect of different fishery mortality scenarios on trophic spectra coming from ET-Transpose (see above). Thus different effort multipliers (Mul_eff variable), ranging conventionally from 0 to 5, are applied to the initial fishing mortalities $F \tau$. Mul_eff $=0$ corresponds 
to a fishery closure $(\mathrm{F}=0)$, and allows users to rebuilt an estimate of the unexploited status of the studied ecosystem. Flow equations enable the calculation of the biomasses $B \tau$, the productions $P \tau$ and the catches $Y \tau$ at the equilibrium for each trophic class and Mul_eff. The other variables contained in the ET_Main table are also treated. We so obtain all the trophic spectra representing the situation at equilibrium for each Mul_eff. Effects of fishery mortality changes at an ecosystem scale include biomass, accessible biomass, and kinetic changes but also impacts on the mean trophic level of the catch and the total biomass. The model provides an overview of the current fishing impact on the ecosystem (compared to the unexploited state), and some long term forecasts on the consequences of increasing or decreasing fishing pressures. This model also enables to see how different ecosystem functioning hypotheses (values of the extent recycling, top-down effect) could affect the ecosystem and trophic scale properties.

ET-Diagnosis is implemented through the create. ETdiagnosis function. It takes as input parameters the list object returned by the create.ETmain function, Mul_eff a vector of the different effort multipliers, and the specific parameters of the ET-diagnosis simulations:

- Beta, a parameter taking values between 0 and 1, which defines the intensity of the biomass recycling by the microbial loop (default value set to 0.1 )

- TopD, a parameter taking values between 0 and 1 , which defines the intensity of the top-down control of predators on their preys (default value set to 0.2 )

- FormD, a parameter taking values between 0 and 1, which defines the functional relationship between preys and predators (default value set to 0.5 )

(cf. package help for more details).

This function returns two types of results for each simulated effort multiplier: indices calculated at the ecosystem scale for each effort multiplier, and the catches, biomasses, accessible biomasses, productions ... for each trophic class.

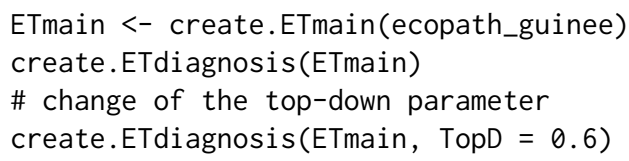

A graphic function, $\operatorname{plot}(m, s c a l e=n u l l$, maxrange=null), displays the principal plots coming from the function create. ETdiagnosis: the biomass, predator biomass, catches ratio plots for the different effort multipliers, and the principle trophic spectra. This function takes as input parameters the list object returned by the create.ETdiagnosis function (m), one scale parameter for the $y$-axis of the BTS (scale, log or not), and the maximum wanted for the x-axis (maxrange). Naturally all the returned graphics are not exhaustive as users can construct other ones using the returned list object. As previously mentioned, we really encourage users to test different scales for the y-axis. Figure 4 is one major graphic of the eight displayed by the function.

\# log scale for the BTS

diag <- create.ETdiagnosis(ETmain)

plot(diag, log)

\section{Summary}

This article describes the principle functions of the EcoTroph package. The package enables the analysis of fishing impacts on aquatic trophic networks in a simple way, and also the simulation of various fisheries in terms of catch (see Figure 1). New simulation tools are currently being developed to improve the model and enable the implementation of more options regarding the fisheries scenarios. The package is available on the Comprehensive R Archive Network (CRAN, http: / /CRAN. R-project . $\mathrm{org} /$ ) and users are encouraged to provide feedback in order to enhance the tool. All the information contained in this article is not exhaustive. More details are available in the package help, and on the website (http://sirs. agrocampus-ouest. fr/EcoTroph). Feel free to contact the authors for any problem encountered while using the package.

\section{Acknowledgement}

We would like to thank all the persons involved in the development of EcoTroph. There are now numerous applications of this model available in the scientific literature and we hope the R package will help to continue to forge ahead. 


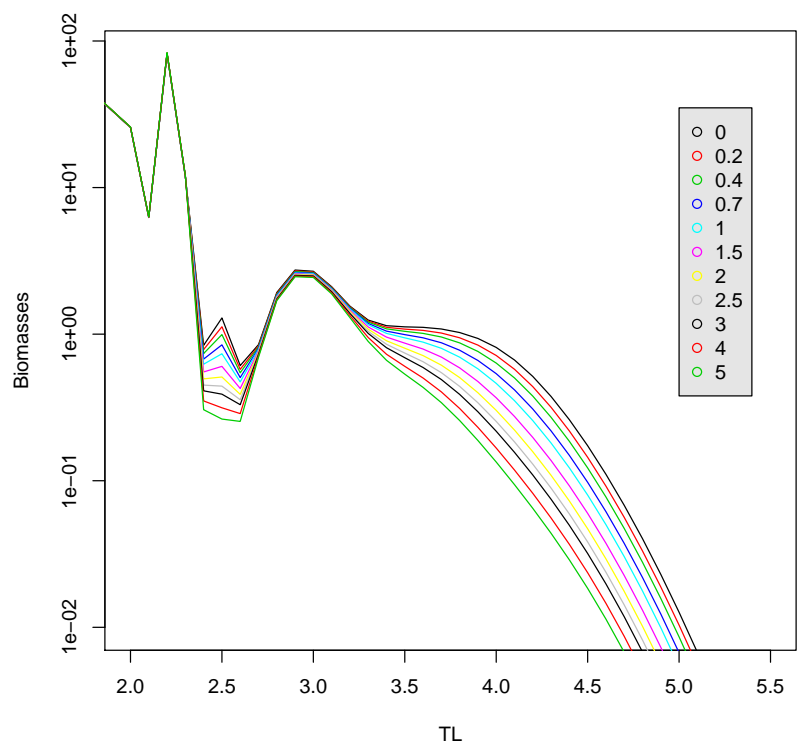

Figure 4: Simulated biomass trophic spectra (BTS) of the Guinean ecosystem for various fishing effort multipliers ranging from 0 (no fishing) to 5 . Such a graph highlights the fishing effects on trophic levels around 2.5 or higher than 3.4, with larger impact for the top predators (highest TLs). In contrast, the low and intermediate ones are less damaged.

\section{Bibliography}

V. Christensen and D. Pauly. ECOPATH II-a software for balancing steady-state ecosystem models and calculating network characteristics. Ecological Modelling, 61(3-4):169-185, 1992. [p98]

M. Colléter, D. Gascuel, J. Ecoutin, and L. Tito de Morais. Modelling trophic flows in ecosystems to assess the efficiency of marine protected area (MPA), a case study on the coast of Sénégal. Ecological Modelling, 232:1-13, 2012. [p98]

C. Elton. Animal Ecology. The Macmillan Company, New York, 1927. [p98]

L. Gasche, D. Gascuel, L. Shannon, and Y. Shin. Global assessment of the fishing impacts on the Southern Benguela ecosystem using an EcoTroph modelling approach. Journal of Marine Systems, 90 (1):1-12, 2012. [p98]

D. Gascuel. The trophic-level based model: A theoretical approach of fishing effects on marine ecosystems. Ecological Modelling, 189(3-4):315-332, 2005. [p98]

D. Gascuel and D. Pauly. EcoTroph: Modelling marine ecosystem functioning and impact of fishing. Ecological Modelling, 220(21):2885-2898, 2009. [p98]

D. Gascuel, Y.-M. Bozec, E. Chassot, A. Colomb, and M. Laurans. The trophic spectrum: Theory and application as an ecosystem indicator. ICES Journal of Marine Science, 62(3):443-452, 2005. [p98]

D. Gascuel, L. Tremblay-Boyer, and D. Pauly. EcoTroph (ET): A trophic level based software for assessing the impacts of fishing on aquatic ecosystems. Fisheries Centre Research Reports, 17(1):1-82, 2009. [p98, 104]

D. Gascuel, S. Guénette, and D. Pauly. The trophic-level-based ecosystem modelling approach: Theoretical overview and practical uses. ICES Journal of Marine Science, 68(7):1403-1416, 2011. [p98, 100]

J. Guitton, M. Colléter, D. Gascuel, and P. Gatti. EcoTroph: EcoTroph modelling support, 2013. URL http://sirs.agrocampus-ouest.fr/EcoTroph/. R package version 1.5-1. [p100]

D. T. Lang. XML: Tools for parsing and generating XML within R and S-Plus., 2012. URL http://CRAN. Rproject. org/package=XML. R package version 3.9-4. [p100] 
G. Lassalle, D. Gascuel, F. Le Loc'h, J. Lobry, G. J. Pierce, V. Ridoux, M. B. Santos, J. Spitz, and N. Niquil. An ecosystem approach for the assessment of fisheries impacts on marine top predators: The Bay of Biscay case study. ICES Journal of Marine Science, 69(6):925-938, 2012. [p98]

R. L. Lindeman. The trophic-dynamic aspect of ecology. Ecology, 23(4):399-417, 1942. [p98]

W. E. Odum and E. J. Heald. The detritus-based food web of an estuarine mangrove community. In L. E. Cronin, editor, Estuarine Research, volume 1, pages 265-286. Academic Press, New York, 1975. [p98]

J. J. Polovina. An overview of the ECOPATH model. Fishbyte, 2(2):5-7, 1984. [p98]

L. Tremblay-Boyer, D. Gascuel, R. Watson, V. Christensen, and D. Pauly. Modelling the effects of fishing on the biomass of the world's oceans from 1950 to 2006. Marine Ecology Progress Series, 442: 169-185, 2011. [p98]

A. Valls, D. Gascuel, S. Guénette, and P. Francour. Modeling trophic interactions to assess the effects of a marine protected area: Case study in the NW Mediterranean Sea. Marine Ecology Progress Series, 456:201-214, 2012. [p98]

Mathieu Colléter

Université Européenne de Bretagne, Agrocampus Ouest, UMR985 ESE, INRA, Agrocampus Ouest

Rennes

France

and

The Fisheries Center, University of British Colombia, Vancouver

Canada

mathieu.colleter@agrocampus-ouest.fr

Jérôme Guitton

Université Européenne de Bretagne, Agrocampus Ouest, UMR985 ESE, INRA, Agrocampus Ouest Rennes

France

jerome.guitton@agrocampus-ouest.fr

Didier Gascuel

Université Européenne de Bretagne, Agrocampus Ouest, UMR985 ESE, INRA, Agrocampus Ouest Rennes

France

didier.gascuel@agrocampus-ouest.fr 